\title{
Effect of Craniocervical Flexion on Muscle Activities of Abdominal and Cervical Muscles During Abdominal Curl-Up Exercise
}

\author{
Tae-lim Yoon ${ }^{1,2}$, MA, PT, Ki-song $\mathrm{Kim}^{3}, \mathrm{PhD}, \mathrm{PT}$ \\ ${ }^{1}$ Applied Kinesiology and Ergonomic Technology Laboratory \\ ${ }^{2}$ Dept. of Physical Therapy, The Graduate School, Yonsei University \\ ${ }^{3}$ Dept. of Physical Therapy, College of Natural Science, Hoseo University
}

\begin{abstract}
Abdominal curl-up exercise may excessively increased superficial neck flexor such as sternocleidomastoid (SCM) muscle. Also, the muscle activity of the abdominal muscles haven't investigated during abdominal curl-up with craniocervical flexion (CCF). Therefore, the purpose of our study was to determine the effect of CCF on the muscle activity of the abdominal and SCM muscles during abdominal curl-up. Twelve healthy subjects (six men and six women) with no history of abdominal or lower back pain within 6 weeks were recruited. Surface electromyographic signals were collected on SCM, rectus abdominis (RA), internal oblique (IO), and external oblique (EO) muscles bilaterally during performing the traditional abdominal curl-up and the abdominal curl-up with CCF. Paired t-tests were used to compare the differences in the muscle activity of the bilateral SCM, RA, EO, and IO muscles between the traditional abdominal curl-up and the abdominal curl-up with CCF $(\mathrm{p}<.05)$. There was significantly lower electromyogram (EMG) activity of the both SCMs during the abdominal curl-up with CCF (Right SCM, 39.50 $\pm 15.29 \%$ MVIC; Left SCM, 38.24 $\pm 17.31 \%$ MVIC) than with the traditional abdominal curl-up (Right SCM, 54.85 $\pm 20.05 \%$ MVIC; Left SCM, 53.18 $\pm 26.72 \%$ MVIC) $(\mathrm{p}<.05$ ). The activity of abdominal muscles were not significantly different between the traditional abdominal curl-up and the abdominal curl-up with CCF. The abdominal curl-up with CCF requires significantly less muscle activity of SCM. Consequently, the abdominal curl-up with CCF is recommended to prevent excessive activation of superficial cervical flexors during abdominal curl-up exercise.
\end{abstract}

Key Words: Deep cervical muscle; Neck pain; Stabilization exercise.

\section{Introduction}

Strong abdominal muscles help stabilize the trunk and lessen lumbar spine stress (Axler and McGill, 1997; Gardner-Morse and Stokes, 1998). Abdominal muscles are usually activated by active flexion of the trunk through a concentric muscle contraction (Escamilla et al, 2006). Trunk flexion occurs during traditional abdominal exercises, such as abdominal curl-up (crunch) or sit-up exercises, as a person raises the head and shoulders off the floor from a supine position toward a sitting position. During the abdominal curl-up exercise, the hips remain at a constant angle and the pelvis does not rotate. In contrast, during the bent-knee or extended-knee sit-up exercise, the hips flex and the pelvis rotates (Ricci et al, 1981). Bent-knee and extended-knee sit-up exercises are effective in activating the rectus abdominis (RA) and external and internal oblique musculature (EO and IO) (Juker et al, 1998). However, the bent-knee sit-up exercise generates greater spinal flexion than the curl-up exercise (Halpern and Bleck, 1979). Therefore, the curl-up exercise may be a safer exercise to perform than the bent knee sit-up exercise for patients required to minimize lumbar spinal flexion because of lumbar

Corresponding author: Ki-song Kim kskim68@hoseo.edu

This research was supported by the Academic Research fund of Hoseo University in 2013 (2013-0046). 
pathologies (Axler and McGill, 1997b).

However, the abdominal curl-up exercise also may activate the hip flexor and lumbar paraspinal muscle, a result that may not be beneficial. For example, high activation levels from the hip flexor and lumbar paraspinal muscles tend to rotate the pelvis anteriorly, increase lumbar lordosis and increase lumbar segmental compression, thus increasing the risk of low back pain (Ralston et al, 1990). Consequently, several studies include designs for increasing isolated abdominal muscle activity without eliciting non-beneficial abdominal muscle activity (Escamilla et al, 2006; Youdas et al, 2008).

Although the prevention of high activation of hip flexor and lumbar erector muscle during abdominal curl-up exercise is a prime cause of low back pain, neck position that cause neck pain also could be a contributing factor of increasing back pain, because vertebral regions are connected with each other, and the condition of each region can be affected by the linked joint structure of the vertebral column (Panjabi, 1992). In chronic neck pain patients, who have weakness of deep cervical stabilizers, may predominantly activate the global muscles of neck such as sternocleidomastoid (SCM) during various tasks and aggravate the neck pain (Armijo-Olivo et al, 2011; Jull et al, 2009; Lindstrøm et al, 2011; O'Leary et al, 2011).

Craniocervical flexion (CCF) is generally recommended to provide craniocervical stability and to $\mathrm{ac}^{-}$ tivate deep cervical stabilizers (Falla et al, 2012; Kelly et al, 2013).

The question of whether the recruiting deep neck muscles with CCF can alter the muscle activity of $\mathrm{RA}, \mathrm{EO}, \mathrm{IO}$, and SCM muscles during abdominal curl-up exercise has not been investigated. Thus this study is designed to determine the effect of CCF on the muscle activity of the abdominal muscles and SCM during abdominal curl-up exercise in healthy subjects. The research hypothesis is that CCF would decrease the muscle activity of SCM and alter the muscle activity of abdominal muscles.

\section{Methods}

\section{Subjects}

Power analysis was performed to estimate the sample size in this study. G-power 3.1.3 software (Franz Faul, Kiel University, Kiel, Germany) was used with an alpha level of .05 , power of .8 , and effect size of .88 , resulting in a sample size requirement of 10. Twelve healthy subjects were recruited to accommodate the calculated sample size. Young healthy subjects (six men and six women) volunteered to participate in this study from a university (Table 1). Individuals were excluded from participation in the study for the following: history of abdominal or lower back pain within six weeks of start of the study or inability to correctly perform the traditional abdominal curl-up and abdominal curl-up with CCF in a pain-free manner (Youdas et al, 2008). Prior to participation, all subjects were informed of the purpose of the study, and informed consent was obtained. This study was approved by Yonsei University Wonju Institutional Review Board.

\section{Instrumentation}

Surface electromyographic (EMG) signals were collected with the Tele-Myo 2400T EMG instrument

Table 1. General characteristics of subjects

\begin{tabular}{cccc}
\hline \hline Variables & Male $\left(\mathrm{n}_{1}=6\right)$ & Female $\left(\mathrm{n}_{2}=6\right)$ & Total $(\mathrm{N}=12)$ \\
\hline Age $($ year $)$ & $22.2 \pm 1.2$ & $21.7 \pm 1.9$ & $21.9 \pm 1.5$ \\
Height $(\mathrm{cm})$ & $175.5 \pm 3.4$ & $163.0 \pm 2.5$ & $154.4 \pm 7.1$ \\
Weight $(\mathrm{kg})$ & $67.0 \pm 3.9$ & $51.0 \pm 3.5$ & $59.0 \pm 9.1$ \\
BMI $^{\mathrm{a}}\left(\mathrm{kg} / \mathrm{m}^{2}\right)$ & $21.8 \pm 1.2$ & $19.2 \pm .9$ & $20.5 \pm 1.7$ \\
\hline
\end{tabular}

body mass index, ${ }^{b}$ mean \pm standard deviation. 
(a wireless telemetry system) (Noraxon, Scottsdale, AZ, USA). EMG data were collected at the sampling rate of $1000 \mathrm{~Hz}$ and analyzed with Myo-Research Master Edition 1.07 XP software (Noraxon, Scottsdale, AZ, USA). The raw signal was filtered using a digital band-pass filter (Lancosh FIR) between 20 and $450 \mathrm{~Hz}$ and notch filtered $(60 \mathrm{~Hz}, 120$ $\mathrm{Hz}$ ). Root-mean-square values were calculated with a moving window of 50milliseconds.

\section{Electrodes}

The skin was prepared before attaching the electrodes by shaving the site and cleaning with alcohol to reduce the skin impedance before positioning the electrodes over each muscle. Disposable $\mathrm{Ag} / \mathrm{AgCl}$ surface electrodes were positioned parallel to the muscle fibers with a center-to-center spacing of 2 $\mathrm{cm}$. All electrodes were placed bilaterally in the following muscles and locations: (a) RA, electrode positioned $2 \mathrm{~cm}$ lateral from the midline of the umbilicus; (b) EO, electrode positioned halfway between the most inferior point of the costal margin of the ribs and the anterior superior iliac spine and angled toward the pubic symphysis in a parallel direction to the fibers of the EO; (c) IO, electrode positioned horizontally within a triangle consisting of a medial border made up of a line from the umbilicus to the pubic symphysis, an inferior border made up of a line from the anterior superior iliac spine (ASIS) to pubis symphysis, and a superior border made up of a line from ASIS to ASIS; and (d) SCM, electrode placed on the muscle belly, at $5 \mathrm{~cm}$ from the mastoid process (Figure 1). Participants were asked to make a brief neck flexion isometric contraction (3-5 s) to confirm that the electrodes had been placed on the right position (Cram and Kasman, 1998; Falla et al, 2002; Guedes et al, 2009; Hermens et al, 2000)

\section{Normalization}

Once the electrodes were attached, EMG data from each muscle tested were collected during a $5 \mathrm{~s}$ maximum voluntary isometric contraction (MVIC). The

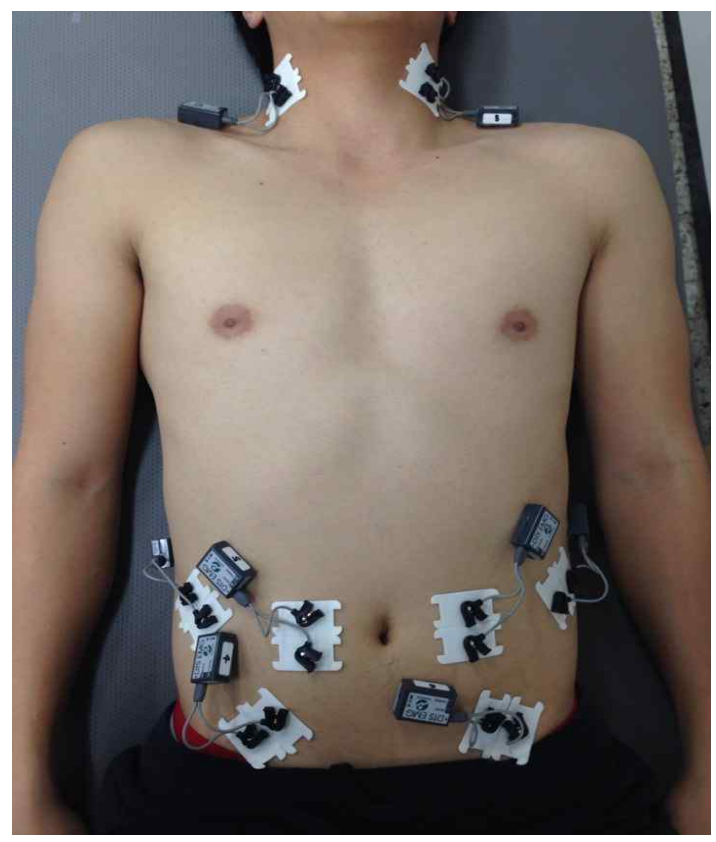

Figure 1. Electrode placements of rectus abdominis, external and internal oblique, and sternocleidomastoid muscles.

MVICs were obtained to normalize the EMG data during the abdominal exercises. For the RA, the subject was positioned supine in a hook-lying position with the feet flat with the thoracolumbar spine fully flexed. The subject's shoulders was forced with manual resistance into trunk extension. For the EO, the subject was instructed in supine with a hook-lying position on the table. The thoracolumbar spine was fully flexed and rotated to the left, with applying manual resistance at the shoulders into extension and right rotation. For the IO muscle, the subject was instructed in supine with a hook-lying position on the table. The thoracolumbar spine was fully flexed and rotated to the right, with applying manual resistance at the shoulders into extension and left rotation (Escamilla et al, 2006; Youdas et al, 2008). For the SCM, a combined movement of craniocervical and cervical flexion in the supine position for $10 \mathrm{~s}$ was used. This movement consists of lifting the head so that it just clears the surface. This position is maintained isometrically with verbal encouragement to maintain a consistent effort during the 
MVIC. Each contraction was repeated three times with a $30 \mathrm{~s}$ rest period between each repetition (Galiano-Castillo et al, 2011).

\section{Procedures}

During familiarization session, each subject received verbal instructions explaining how to correctly perform the traditional abdominal curl-up and the abdominal curl-up with CCF. For familiarization with the traditional abdominal curl-up, the subject was instructed to lay supine with both arms down by the side, the hands relaxed, the knees flexed approximately 90 degrees, the hips flexed approximately 45 degrees, and the feet placed flat on the table (Escamilla et al, 2006). An investigator held the legs of subject to prevent altering the position during the traditional abdominal curl-up exercise. Subjects elevated the trunk by lifting the head and shoulders while staring straight ahead such that the scapulae were lifted above the table. We installed a target bar that could be positioned to the chest at the point when the scapulae came off the ground (Figure 2A). This was to ensure that both scapulae were consistently elevated above the table for each abdominal curl-up exercise. The subject was asked to touch the target bar with each abdominal curl-up exercise in 2 $\mathrm{s}$ and hold the position for $3 \mathrm{~s}$ (Hildenbrand and Noble, 2004).

For familiarization with the abdominal curl-up

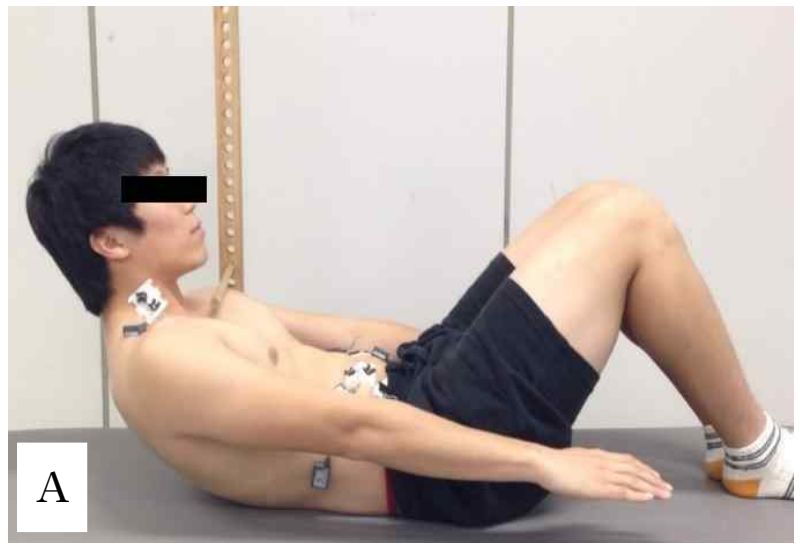

with $\mathrm{CCF}$, subjects learned how to perform CCF first. The subject begins in a supine hook-lying $\mathrm{po}^{-}$ sition, hands resting by side. With instruction, the subject raises the head just enough to allow a investigator to slide the widths of the index and middle finger of one hand, one atop the other, under the subject's head at the most posterior aspect of the occiput. The subject is asked to relax their neck, resting their head on the examiner's fingers. During the practice, the investigator gently moves his/her fingers side to side under the subject's head, providing a tactile cue for maintaining proper head position above the plinth (Olson et al, 2006). When the CCF is correctly performed, a skin fold is produced posterior to the mandible. Subjects next practiced the $\mathrm{ab}^{-}$ dominal curl-up with CCF. During the abdominal curl-up with CCF, loss of CCF was determined by observing the skin fold produced posterior to the mandible when the head was maximally flexed on the neck by investigator (Figure 2B). When the fold began to open, the subject was given the verbal cue for correction the neck position by investigator.

Once the subject correctly performed the traditional abdominal curl-up and the abdominal curl-up with CCF, a testing session was scheduled after an hour of rest. During data collection, all subjects performed the traditional abdominal curl-up and the $a b^{-}$ dominal curl-up with CCF under the close supervision of investigator. When the subject's chest

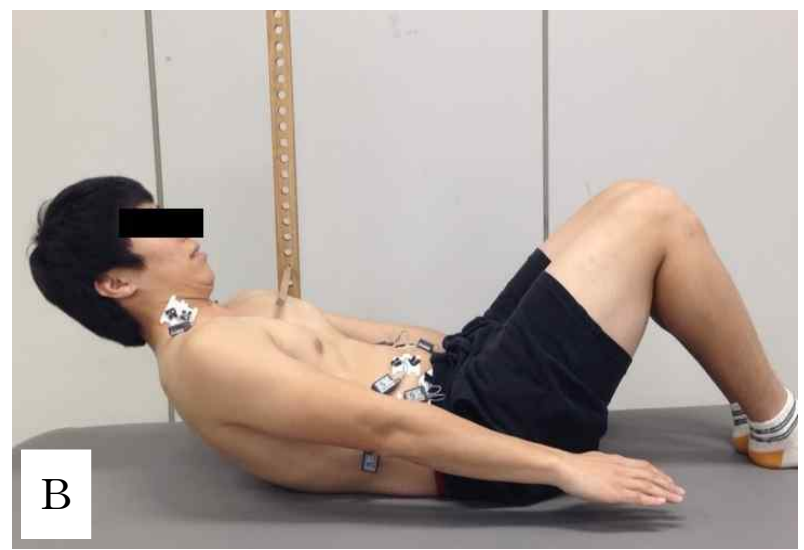

Figure 2. Curl up (A: traditional abdominal curl-up, B: Abdominal curl-up with craniocervical flexion). 
touched the target bar, the subject isometrically held the position for a count of $3 \mathrm{~s}$. This process was repeated for to average the data. A two minute rest was given between each exercise trial to prevent muscle fatigue. The performing order of the traditional abdominal curl-up and the abdominal curl-up with CCF was randomly assigned by drawing lots. This randomization was to minimize threats to the study's internal validity (Youdas et al, 2008).

\section{Statistical analysis}

We used Kolmogorov-Smirnov $Z$ to test whether continuous data approximated a normal distribution, and paired-t tests to compare the differences in normalized EMG muscle activity of the bilateral SCM, $\mathrm{RF}, \mathrm{EO}$, and IO muscles (dependent variable) between the traditional abdominal curl-up and the abdominal curl-up with CCF (independent variable). We rejected null hypotheses of no difference if $\mathrm{p}^{-}$-values were less than .05, and used SPSS ver. 18.0 software for all data analysis (SPSS, Inc., Chicago, IL, USA).

\section{Results}

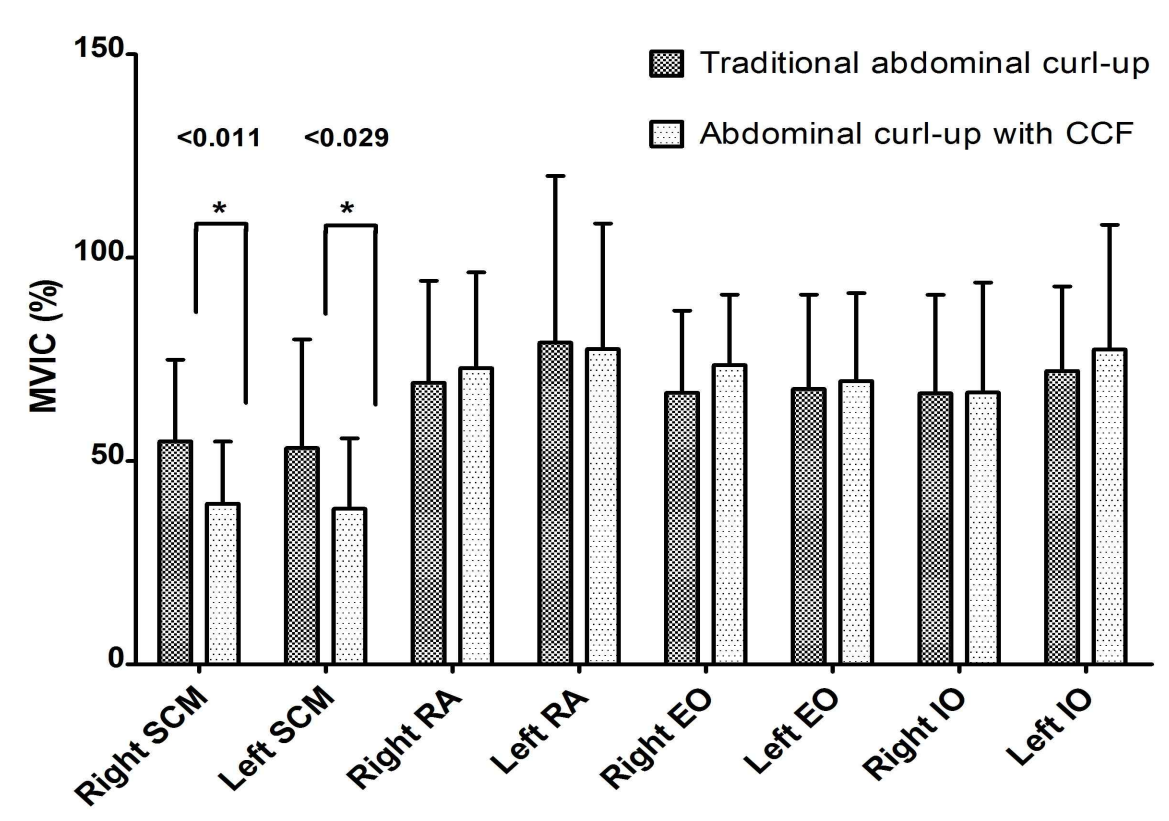

All continuous variables approximate a normal distribution (Kolmogorov-Smirnov $Z$ test, $\mathrm{p}>$.05). There was significantly lower EMG activity of the both SCMs during the abdominal curl-up with CCF (Right SCM, 39.50 $\pm 15.29 \%$ MVIC; Left SCM, $38.24 \pm 17.31 \% \mathrm{MVIC})$ than with the traditional abdominal curl-up (Right SCM, 54.85 $\pm 20.05 \%$ MVIC; Left SCM, 53.18 $\pm 26.72 \%$ MVIC $) \quad(\mathrm{p}<.05)$. The changes of the activity of abdominal muscles in the abdominal curl-up exercise with CCF were not significantly different from the traditional abdominal curl-up exercise, but most means of the \%MVIC of abdominal muscles in abdominal curl-up exercise with CCF were slightly greater than that in the traditional $\mathrm{ab}^{-}$ dominal curl-up exercise except left RA (Figure 3).

\section{Discussion}

The aim of this study was to investigate the effects of CCF on the muscle activity of abdominal muscles (RA, IO and EO) and SCM during two dif-

Figure 3. Comparing traditional abdominal curl-up and abdominal curl-up with craniocervical flexion (SCM: sternocleidomastoid, RA: rectus abdominis, IO: internal oblique, EO: external oblique, MVIC: maximum voluntary isometric contraction). 
ferent abdominal curl-up exercises. The results of this study showed that CCF decreased the muscle activity of SCM significantly. However, CCF did not alter the activation pattern of the muscle activity of abdominal muscles significantly. Thus the findings of this study partially supported the research hypothesis.

The muscle activity of SCM during abdominal curl-up exercise with CCF was significantly lower

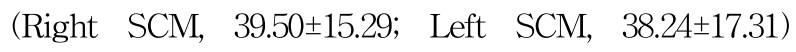
than that during the traditional abdominal curl-up exercise (Right SCM, 54.85 \pm 20.05 ; Left SCM, 53.18 \pm 26.72 ). This result is consistent with previous studies, which reported the role of the CCF in preventing excessive activation of global muscle during functional activity (Falla et al, 2012; Kelly et al, 2013; Kim et al, 2012). We chose abdominal curl up exercise as a test exercise because people with neck pain report greater activation of SCM muscles and complain of neck discomfort or even cervical pain associated with abdominal curl up exercise (Falla et al, 2004; Galiano-Castillo et al, 2011; Nederhand et al, 2002).

Thus, we suggest performing the CCF to prevent cervical discomfort during abdominal curl up exercise. In addition, considering the significantly reduced bilateral SCM activity, CCF may delay the fatigue and hypertrophy of SCM that occurs after repetitive functional activity or exercise (Ylinen et al, 2003). Repetitive contraction of SCM can lead to muscle fatigue due to Type II fiber composition increment and localized muscle fatigue (Ang et al, 2005). Decreased muscle pulling action of SCM can alleviate the compressive force in cervical spinal column and activate the deep cervical flexors such longus colli, longus capitis, and rectus capitis anterior and lateralis (Moroney et al, 1988; Siegmund et al, 2007). However, the effect of the CCF in stabilizing the cervical area by stiffening the cervical spinal column and facilitating local muscles could not be assessed in this study.

Contrary to our research hypothesis, CCF did not influence the abdominal muscles activity during abdominal curl-up exercise. However, although the changes of the activity of abdominal muscles in abdominal curl-up exercise with CCF were not significantly greater than that in the traditional abdominal curl-up exercise, most means of the \%MVIC of abdominal muscles in abdominal curl-up exercise with $\mathrm{CCF}$ were greater than that in the traditional abdominal curl-up exercise except left RA. The increased \%MVIC of the abdominal muscles in abdominal curl-up exercise with CCF could be caused by reduced cervical curve flexion due to decreased SCM activity. A previous study claimed that CCF minimized the activation of superficial cervical muscles (Jull et al, 2008). This reduced cervical flexion in abdominal curl-up exercise with CCF could increase the abdominal muscles activity from lengthened lever arm of trunk while performing abdominal curl-up exercise. Moreover, subjects in this study were young and healthy. Subjects with weak abdominal muscles could have activated abdominal muscles during abdominal curl-up exercise with CCF.

This study has several limitations. First, because healthy young subjects performed curl-up exercise with two conditions, generalizability is limited. Second, kinematic data of cervical position while performing curl-up was not obtained. Thus, the CCF position was only confirmed with investigators observation. Further study with a kinematic analysis of a patient population is warranted to generalize the findings of this study.

\section{Conclusion}

The abdominal curl-up with CCF requires significantly less muscle activity of SCM than the traditional curl-up. There was no differences \%MVIC of abdominal muscles between the abdominal curl-up with CCF and the traditional curl-up. Therefore, the abdominal curl-up with $\mathrm{CCF}$ is recommend to pre- 
vent excessive activation of superficial cervical flexors during abdominal curl-up exercise.

\section{References}

Ang B, Linder J, Harms-Ringdahl K. Neck strength and myoelectric fatigue in fighter and helicopter pilots with a history of neck pain. Aviat Space Environ Med. 2005;76(4):375-380.

Armijo-Olivo S, Silvestre R, Fuentes J, et al. Electromyographic activity of the cervical flexor muscles in patients with temporomandibular disorders while performing the craniocervica flexion test: A cross-sectional study. Phys Ther. 2011; 91(8):1184-1197.

Axler CT, McGill SM. Low back loads over a variety of abdominal exercises: Searching for the safest abdominal challenge. Med Sci Sports Exerc. 1997a;29(6):804-811.

Axler CT, McGill SM. Low back loads over a variety of abdominal exercises: Searching for the safest abdominal challenge. Med Sci Sports Exerc. 1997b;29(6):804-811.

Cram J, Kasman G. Introduction to Surface Electromyography. 1st ed. Gaithersburg, MD, Aspen Publishers, Inc., 1998:279-349.

Escamilla RF, McTaggart MS, Fricklas EJ, et al. An electromyographic analysis of commercial and common abdominal exercises: Implications for rehabilitation and training. J Orthop Sports Phys Ther. 2006;36(2):45-57.

Falla D, Bilenkij G, Jull G. Patients with chronic neck pain demonstrate altered patterns of muscle activation during performance of a functional upper limb task. Spine (Phila Pa 1976). 2004;29(13):1436-1440.

Falla D, Dall'Alba P, Rainoldi A, et al. Location of innervation zones of sternocleidomastoid and scalene muscles--a basis for clinical and $\mathrm{re}^{-}$ search electromyography applications. Clin Neurophysiol. 2002;113(1):57-63.
Falla D, O'Leary S, Farina D, et al. The change in deep cervical flexor activity after training is associated with the degree of pain reduction in patients with chronic neck pain. Clin J Pain. 2012;28(7):628-634.

Galiano-Castillo N, Fernández-Lao C, CantareroVillanueva I, et al. Altered pattern of cervical muscle activation during performance of a functional upper limb task in breast cancer survivors. Am J Phys Med Rehabil. 2011;90(5): 349-355.

Gardner-Morse MG, Stokes IA. The effects of abdominal muscle coactivation on lumbar spine stability. Spine (Phila Pa 1976). 1998;23(1):86-91.

Guedes LU, Parreira VF, Diório AC, et al. Electromyographic activity of sternocleidomastoid muscle in patients with parkinson's disease. J Electromyogr Kinesiol. 2009;19(4):591-597.

Halpern AA, Bleck EE. Sit-up exercises: An electromyographic study. Clin Orthop Relat Res. 1979; (145):172-178.

Hermens HJ, Freriks B, Disselhorst-Klug C, et al. Development of recommendations for semg sensors and sensor placement procedures. J Electromyogr Kinesiol. 2000;10(5):361-374.

Hildenbrand K, Noble L. Abdominal muscle activity while performing trunk-flexion exercises using the ab roller, abslide, fitball, and conventionally performed trunk curls. J Athl Train. 2004;39 (1):37-43.

Juker D, McGill S, Kropf P, et al. Quantitative intramuscular myoelectric activity of lumbar portions of psoas and the abdominal wall during a wide variety of tasks. Med Sci Sports Exerc. 1998;30 (2):301-310.

Jull GA, O'Leary SP, Falla DL. Clinical assessment of the deep cervical flexor muscles: The craniocervical flexion test. J Manipulative Physiol Ther. 2008;31(7):525-533.

Jull GA, Falla D, Vicenzino B, et al. The effect of therapeutic exercise on activation of the deep cervical flexor muscles in people with chronic 
neck pain. Man Ther. 2009;14(6):696-701.

Kelly M, Cardy N, Melvin E, et al. The craniocervical flexion test: An investigation of performance in young asymptomatic subjects. Man Ther. 2013;18(1):83-86.

Kim K, Lee G, Cynn H, et al. Electromyographic analysis of thoracic and lumbar erector spinae activity using the abdominal drawing-in maneuver and chin tuck during prone thoracic $\mathrm{ex}^{-}$ tension exercises. Phys Ther Kor. 2012;19(4):1-7.

Lindstrøm R, Schomacher J, Farina D, et al. Association between neck muscle coactivation, pain, and strength in women with neck pain. Man Ther. 2011;16(1):80-86.

Moroney SP, Schultz AB, Miller JA. Analysis and measurement of neck loads. J Orthop Res. 1988;6(5):713-720.

Nederhand MJ, Hermens HJ, IJzerman MJ, et al. Cervical muscle dysfunction in chronic whiplash-associated disorder grade 2: The relevance of the trauma. Spine (Phila Pa 1976). 2002;27 (10):1056-1061.

O’Leary S, Falla D, Jull G. The relationship between superficial muscle activity during the cranio-cervical flexion test and clinical features in patients with chronic neck pain. Man Ther. 2011;16(5):452-455.

Olson LE, Millar AL, Dunker J, et al. Reliability of a clinical test for deep cervical flexor endurance. $\mathrm{J}$
Manipulative Physiol Ther. 2006;29(2):134-138. Panjabi MM. The stabilizing system of the spine. Part i. Function, dysfunction, adaptation, and enhancement. J Spinal Disord. 1992;5(4):383-389.

Ralston SH, Urquhart GD, Brzeski M, et al. Prevalence of vertebral compression fractures due to osteoporosis in ankylosing spondylitis. BMJ. 1990;300(6724):563-565.

Ricci B, Marchetti M, Figura F. Biomechanics of sit-up exercises. Med Sci Sports Exerc. 1981;13 (1):54-59.

Siegmund GP, Blouin JS, Brault JR, et al. Electromyography of superficial and deep neck muscles during isometric, voluntary, and reflex contractions. J Biomech Eng. 2007;129(1):66-77.

Ylinen J, Takala EP, Nykänen M, et al. Active neck muscle training in the treatment of chronic neck pain in women: A randomized controlled trial. JAMA. 2003;289(19):2509-2516.

Youdas JW, Guck BR, Hebrink RC, et al. An electromyographic analysis of the ab-slide exercise, abdominal crunch, supine double leg thrust, and side bridge in healthy young adults: Implications for rehabilitation professionals. J Strength Cond Res. 2008;22(6):1939-1946.

This article was received September 10, 2013, was reviewed September 10, 2013, and was accepted October 31, 2013. 